Polymer Journal, Vol. 22, No. 11, pp 969-975 (1990)

\title{
Preparation of Styrene Derivatives Containing Sulfide Group: Addition Reactions of Thiophenol and Its Derivatives to 1,4-Divinylbenzene
}

\author{
Eiichi Kobayashi, * Jian Jiang, Hiroyoshi Matsumoto, \\ and Junji FURUKaWA \\ Department of Industrial Chemistry, Faculty of Science and Technology, \\ Science University of Tokyo, Noda, Chiba 278, Japan
}

(Received April 13, 1990)

\begin{abstract}
To prepare styrene derivatives containing a sulfide group such as 4-vinyl-1[(2-phenylthio)ethyl] benzenes, the addition reactions of thiophenols to 1,4-divinylbenzene were carried out in the presence of AIBN or BPO. The maximum yields of the corresponding adducts were about $62 \%$ by the G.C. method. The competitive addition reaction of thiophenol to 1,4divinylbenzene and the remaining vinyl group of the mono-adduct simultaneously took place to decrease the yield of the mono-adduct. The effects of the reaction time, reaction temperature and the additive in the course of the reaction are discussed based on gas chromatographic studies. The monomer reactivity ratio of the obtained mono-adduct was estimated by the radical copolymerization with MMA.
\end{abstract}

KEY WORDS Addition Reaction / Thiophenol / 1,4-Divinylbenzene / p-Arylthioalkylstyrene / 4-Vinyl-1-[(2-phenylthio)ethyl]benzene / 4-Vinyl1-[( $p$-tolylthio $)$ ethyl $]$ benzene / 4-Vinyl-1-[( $m$-tolylthio $)$ ethyl $]$ benzene / Copolymerization / Monomer Reactivity Ratio /

Many kinds of styrene derivatives containing characteristic functional groups have been synthesized and used to prepare new advanced materials. For instance, Tsuruta et al. ${ }^{1-3}$ studied the addition reactions of various amines to divinylbenzene by a lithium alkylamide catalyst to prepare styrene derivatives containing amine groups. The copolymerization with styrene was carried out to prepare copolymers with a sea-island microstructure or a lamellar microdomain which was found to be effective for antithrombogenic materials. Nakahama et al..$^{4-5}$ reported also the preparation of styrene derivatives containing silyl groups. The silyl group was used to protect the reactive functional groups in the monomers toward anionic living polymerization of styrenes. Asahara et al. ${ }^{7}$ prepared al- kylthiomethylstyrene or arylthiomethylstyrene by a condensation reaction of chloromethylstyrene with sodium alkyl sulfide or sodium phenyl sulfide. The polymers composed of the sulfide monomers were highly reactive against an electron beam to develop a positive pattern. ${ }^{8}$

On the other hand, the addition reaction of a thiol group to an unsaturated carbon-carbon bond, which yields anti-Markownikoff type adducts in the presence of a radical initiator, is well known. ${ }^{9-11}$ Screttas et al. carried out the addition reaction of thiophenol to olefin and diolefin to synthesize sulfide derivatives. ${ }^{12}$ Another kinetic approach to the addition reaction of thiophenol to styrene showed ${ }^{13,14}$ that the reaction rate depends on the concentration of thiophenol. The rate determining step is the hydrogen transfer from

* To whom all correspondence should be addressed. 
thiophenol to the intermediate carbon radical. However, few investigations have been conducted to prepare new styrene derivatives by the addition reaction.

The authors developed a new synthetic method for styrene derivatives containing a sulfide group by the addition reaction of thiophenol (TP) and its derivatives to 1,4divinylbenzene (DVB) (Scheme 1).

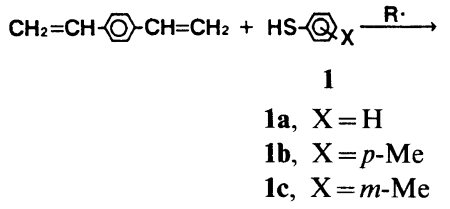

Scheme 1.

In this paper, the authors deal with (i) synthesis of styrene derivatives containing a sulfide group, $\mathbf{2 a}, \mathbf{2 b}$, and $\mathbf{2 c}$, (ii) elucidation of the reaction mechanism, and (iii) the copolymerization of $\mathbf{2 a}$ with methyl methacrylate and the estimation of $Q$ and $e$ values of the monomer $\mathbf{2 a}$.

\section{RESULTS AND DISCUSSION}

\section{Addition Reactions of Thiophenol and Its Derivatives to $D V B$}

Addition reaction mechanisms of thiophenols to styrenes are already examined in this laboratory. ${ }^{15}$ Here, addition reactions of thiophenols to DVB are extensively studied to obtain $\mathbf{2 a}, \mathbf{2 b}$, and $\mathbf{2 c}$. To synthesize new styrene derivatives containing a sulfide group, an addition reaction of 1 a with DVB was carried out at $75^{\circ} \mathrm{C}$ in benzene in the presence of small amount of initiators such as AIBN and BPO. An equimolar amount of $1 \mathrm{a}$ and DVB with a small amount of the radical initiator was charged into a $50 \mathrm{ml}$ round bottom flask

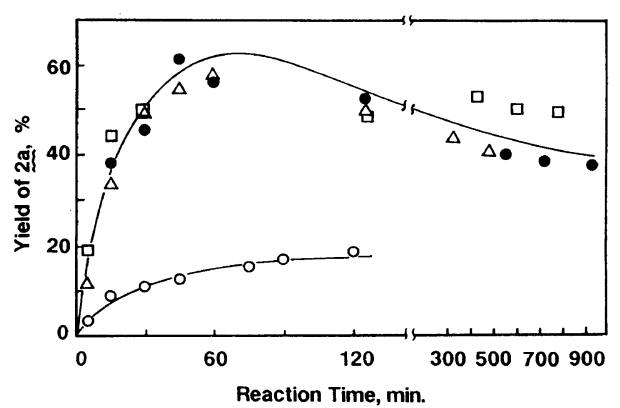

Figure 1. Relationship between reaction time and yield of $2 \mathrm{a}$ in benzene at $75^{\circ} \mathrm{C}$ under nitrogen atmosphere. $[\mathrm{DVB}]_{0}=[1 \mathrm{a}]_{0}=0.34 \mathrm{moll}^{-1} ; \quad[$ Initiator $]=2.0 \times 10^{-3}$ mol1 $1^{-1}$., AIBN; $\triangle$, AIBN plus $1.87 \mathrm{ppm}$ oxygen added against total monomer; $\square$, BPO; $O$, without initiator.

equipped with a three-way stopcock under nitrogen atmosphere. The reaction solution was colorless transparent from the beginning to the end of the reaction.

As shown in Figure 1, the yield of $\mathbf{2 a}$ measured by G.C. method was significantly affected by the initiation mode. In the presence of the radical initiators, AIBN and BPO, the yield of 2 a rapidly increased up to about $65 \%$, however, it remained about $20 \%$ without adding any initiator. A small amount of oxygen initiated the addition reaction, but a large amount of oxygen decreased the yield of $2 \mathbf{a}$ as discussed later. The yield of $2 \mathbf{a}$ reached a maximum of about $62 \%$ for $60 \mathrm{~min}$, then decreased gradually with reaction time. After the reaction was stopped, 2 a was isolated with two methods, column chromatography or distillation under reduced pressure. For details, see EXPERIMENTAL. The isolated yield of 2a was about $50 \%$.

${ }^{1} \mathrm{H}$ NMR spectrum of the isolated $2 \mathbf{a}$ is shown in Figure 2. There appeared three main peaks, characterized as absorptions of benzene rings $(6.7-7.1 \mathrm{ppm}, 9 \mathrm{H})$, vinyl goup (4.9-6.6 ppm, $3 \mathrm{H})$ and methylene $(2.6-3.2 \mathrm{ppm}, 4 \mathrm{H})$ of 2a. In addition, no signals assignable to other structures than $\mathbf{2 a}$ could be detected; e.g., no absorption peaks at $1.5 \mathrm{ppm}$ and $3.5 \mathrm{ppm}$ assigned for the methylene and methine of polymers of DVB and 2a, respectively. In the 


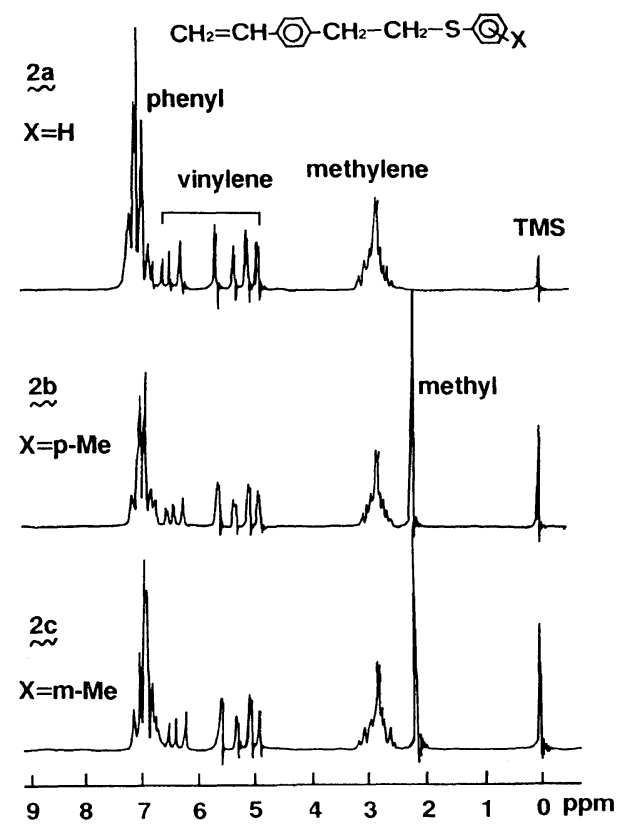

Figure 2. ${ }^{1} \mathrm{H}$ NMR spectra of $\mathbf{2 a}, \mathbf{2 b}$ and $\mathbf{2 c}$, in $\mathrm{CDCl}_{3}$. TMS as standard.

${ }^{1} \mathrm{H}$ NMR spectra of $\mathbf{2 b}$ and $2 \mathrm{c}$, besides the absorptions of benzene rings $(6.7-7.1 \mathrm{ppm})$, vinyl group (4.9-6.6 ppm), and methylene (2.6-3.2 ppm), the characteristic methyl peaks appear at $2.2 \mathrm{ppm}$.

Figure 3 shows the IR spectrum of $2 \mathbf{a}$. The characteristic absorption peaks of the methylene group at 2940 and $2850 \mathrm{~cm}^{-1}$, the strong absorption peaks of the vinyl group at 1630 , 980 , and $900 \mathrm{~cm}^{-1}$, as well as the $p$-substituted and mono-substituted benzene rings appear at 820 and $730 \mathrm{~cm}^{-1}$, respectively. With the comparison of the observed sulfur content of 2a, $13.3 \%$ (Calcd $13.3 \%$ ), it is clearly explained that the addition reaction of 1 a to DVB is capable of producing a novel styrene derivative containing the sulfide group, 2a. In the IR spectra of $\mathbf{2 b}$ and $\mathbf{2 c}$, besides the absorption peaks appeared in the IR spectrum of $\mathbf{2 a}$, methyl absorptions newly appear at 2995 and $1380 \mathrm{~cm}^{-1}$.

The addition reaction of $1 \mathrm{a}$ to DVB took place in the radical chain mechanism shown in Scheme 2, where I means a radical initiator.

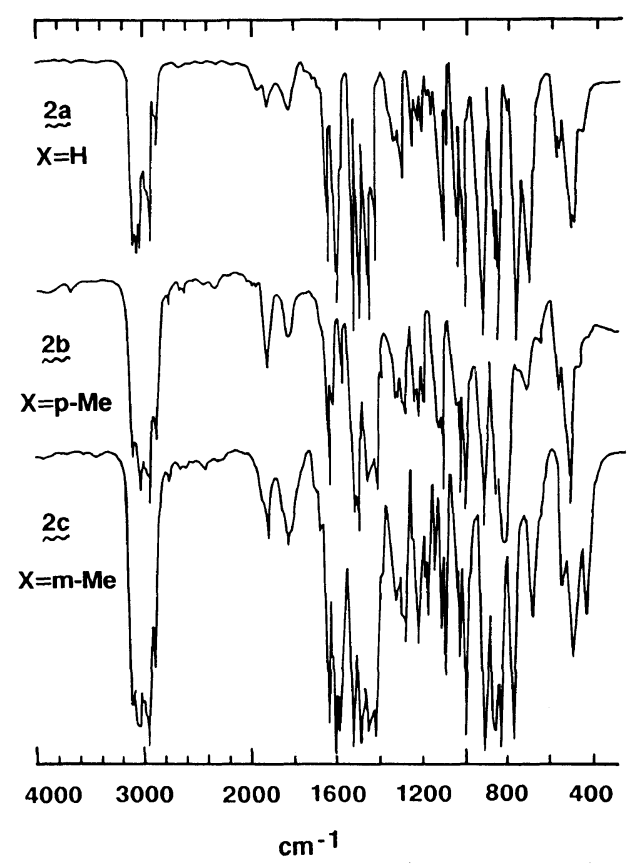

Figure 3. IR spectra of $\mathbf{2 a}, \mathbf{2 b}$, and $\mathbf{2 c}$. Liquid film method.

$$
\begin{aligned}
& \mathrm{I} \stackrel{k_{d}}{\longrightarrow} 2 \mathrm{R} . \\
& \mathrm{R} \cdot+\mathrm{TP} \stackrel{\mathrm{k}_{\mathrm{i}}}{\longrightarrow} \phi \mathrm{S} \cdot+\mathrm{RH} \\
& \phi \mathrm{S} \cdot+\mathrm{DVB} \stackrel{\mathrm{ka}_{\mathrm{a} 1}}{\longrightarrow} \mathrm{CH}_{2}=\mathrm{CH} \phi \dot{\mathrm{C}} \mathrm{H}-\mathrm{CH}_{2}-\mathrm{S}-\phi \\
& \mathrm{CH}_{2}=\mathrm{CH} \phi \dot{\mathrm{C}} \mathrm{HCH}_{2} \mathrm{~S} \phi+\mathrm{TP} \stackrel{\mathrm{ktr}_{\mathrm{t}}}{\longrightarrow} \mathrm{CH}_{2}=\mathrm{CH} \phi \mathrm{CH}_{2} \mathrm{CH}_{2} \mathrm{~S} \phi+\phi \mathrm{S} .
\end{aligned}
$$$$
\text { 2a }
$$

Scheme 2.

With the repetition of reactions (3) and (4), the product $\mathbf{2 a}$ was produced successively by the chain reaction. The reaction scheme is already discussed based on the kinetic method. ${ }^{16}$ However, the product $2 \mathrm{a}$ possesses the reactive vinyl group toward thiophenol 1a and active species for the polymerization. Accordingly, DVB simultaneously turns into products 2a and 3a as shown in Scheme 3. Indeed, the addition reaction of two moles of 1 a with one mole of DVB was reported to give the 3a quantitatively. ${ }^{12}$ Polymerization of DVB and $2 \mathbf{a}$ would reduce also the yield of $\mathbf{2 a}$, if it occurred. However, only a small amount of polymers was recovered, since thiophenol can 
act as an effective chain transfer reagent to prevent polymerizations. ${ }^{17}$ Consequently, the formation of the di-adduct 3a may be the main factor to reduce the yield of the mono-adduct 2a.

$$
\phi \mathrm{S} \cdot+2 \mathbf{a} \stackrel{\mathbf{k}_{\mathbf{a} 2}}{\longrightarrow} \phi \mathrm{SCH}_{2} \dot{\mathrm{C}} \mathrm{H} \phi \mathrm{CH}_{2} \mathrm{CH}_{2} \mathrm{~S} \phi
$$

$\phi \mathrm{SCH} \mathrm{C}_{2} \dot{\mathrm{C}} \phi \mathrm{CH}_{2} \mathrm{CH}_{2} \mathrm{~S} \phi+\mathrm{TP} \stackrel{k_{\mathrm{tr} 2}}{\longrightarrow} \phi \mathrm{SCH}_{2} \mathrm{CH}_{2} \phi \mathrm{CH}_{2} \mathrm{CH}_{2} \mathrm{~S} \phi+\phi \mathrm{S}$.

3a

Scheme 3.

The thiophenol derivatives, $p$-toluenethiol and $m$-toluenethiol also reacted with DVB to give the corresponding novel adducts, $\mathbf{2} \mathbf{b}$ and $2 \mathrm{c}$ with about $50 \%$ of the isolated yield. These addition reactions behave quite the same as that of $1 \mathbf{a}$ with DVB. ${ }^{1} \mathrm{H}$ NMR spectra of $\mathbf{2 b}$ and $\mathbf{2 c}$ are almost equal to that of $\mathbf{2 a}$, except the presence of the methyl group in the tolyl group at $2.2 \mathrm{ppm}$ as already shown in Figure 2. The IR spectra showed characteristic absorption peaks of vinyl groups at 1630$1625 \mathrm{~cm}^{-1}$, and the methyl and methylene groups at 2995, 2920, and $2850 \mathrm{~cm}^{-1}$ (Figure 3). From these spectroscopic observations, as well as the sulfur content of $\mathbf{2 b}$ and $\mathbf{2 c}$, it is realized that the structures of $\mathbf{2 b}$ and $\mathbf{2 c}$ are similar as those shown in Scheme 1.

\section{Effect of Reaction Temperature}

Figure 4 shows the effect of the reaction temperature on the addition reaction of 1a with DVB under nitrogen atmosphere. The maximum yield of 2a depends clearly on the reaction temperature, which is attained about $65 \%$ at $75^{\circ} \mathrm{C}$ for $50 \mathrm{~min}$. However, the reaction time giving the maximum yields becomes longer with decrease of the reaction temperature. To obtain the maximum yield in a relatively short reaction time, it is necessary that the addition reaction of 1 a with DVB may be carried out at a high temperature, such as $75^{\circ} \mathrm{C}$.

According to the temperature dependence of the initial reaction rate shown in Figure 4, the

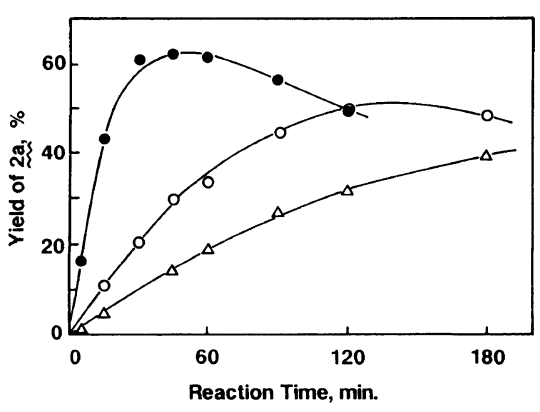

Figure 4. Relationship between yield of 2a and reaction temperature in benzene under nitrogen atmosphere. $75^{\circ} \mathrm{C} ; \mathrm{O}, 65^{\circ} \mathrm{C} ; \triangle, 55^{\circ} \mathrm{C} ;[\mathrm{DVB}]_{0}=[1 \mathrm{a}]_{0}=0.34 \mathrm{moll}^{-1}$; $[\mathrm{AIBN}]=2.0 \times 10^{-3} \mathrm{moll}^{-1}$.

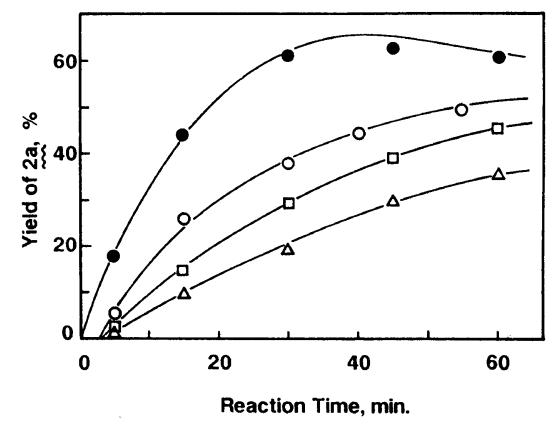

Figure 5. Effect of oxygen on the yield of $2 \mathbf{a}$ in benzene under air atmosphere: $\bigcirc, 75^{\circ} \mathrm{C} ; \square, 65^{\circ} \mathrm{C}, \triangle, 55^{\circ} \mathrm{C}$; $\bigcirc$, $75^{\circ} \mathrm{C}$ under nitrogen atmosphere. $[\mathrm{DVB}]_{0}=[1 \mathrm{a}]_{0}=0.34$ $\mathrm{mol} \mathrm{l}{ }^{-1} ;[\mathrm{AIBN}]=2.0 \times 10^{-3} \mathrm{moll}^{-1}$.

apparent activation energy of the addition reaction of 1a with DVB was estimated to be about $30 \mathrm{kcal} \mathrm{mol}^{-1}$, which is almost the same as the activation energy of the decomposition of AIBN (about $31 \mathrm{kcal} \mathrm{mol}^{-1}$ ). ${ }^{18}$ It seems that the reaction rate may be controlled by a decomposition rate of the radical initiator, so that the high reaction temperature would accelerate the addition reaction giving relatively high yield in a short reaction time.

\section{Effect of Oxygen}

It is generally believed that oxygen retards a radical chain reaction as a radical inhibitor. Figure 5 shows the relationship between the reaction time and the yield of $2 \mathbf{a}$ in the addition reaction of 1a with DVB under air at 55 to $75^{\circ} \mathrm{C}$ or under nitrogen atmosphere at $75^{\circ} \mathrm{C}$. 
In the presence of oxygen, a short induction period appears. The reaction rate under nitrogen is faster than that under air, since oxygen in air could disturb the chain reaction mentioned in Scheme 1. On the other hand, compared with the addition reaction under nitrogen atmosphere shown in Figure 4, at the relatively low temperature, e.g., at $65^{\circ} \mathrm{C}$ and $55^{\circ} \mathrm{C}$, the reaction rates obtained under air became higher than those under nitrogen atmosphere.

Actually, at a relatively low temperature oxygen oxidates mercaptan to form disulfide. ${ }^{19}$ In the presence of vinyl compounds, the intermediate thiyl radical attacks vinyl compounds giving adducts. Oxygen may accelerate the chain reaction involving thiols even at the low temprature.

\section{Effect of Additive}

Since vinyl groups of 2a and DVB are able to participate in the homo- and copolymerization to reduce the yield of $2 \mathbf{a}$, a small amount of the additive as a polymerization inhibitor, $p$-tert-butylcatechol was added in the reaction mixture. Figure 6 shows the effects of the addition of $p$-tert-butylcatechol on the reaction.

When p-tert-butylcatechol was added in the amount of 100 to $2000 \mathrm{ppm}$, the reaction rate forming $\mathbf{2 a}$ decreased, as well as the yield of 2a also decreased below $60 \%$, but no homo- and copolymers were detected. If p-tert-butylcatechol was not added to the reaction mixture, less than $5 \%$ of oligomers would formed in benezene at $60^{\circ} \mathrm{C}^{20} \mathrm{In}$ general, catechols stop markedly radical polymerizations of vinyl compounds. However, p-tert-butylcatechol does not affect so much the addition reaction of thiophenol to DVB, since the carbon radical probably reacts to both p-tert-butylcatechol and thiophenol. The reactivity of $p$-tert-butylcatechol is lower than that of thiophenol. The p-tert-butylcatechol radical is a stable one and may abstract hydrogen from thiophenol. Consequently,

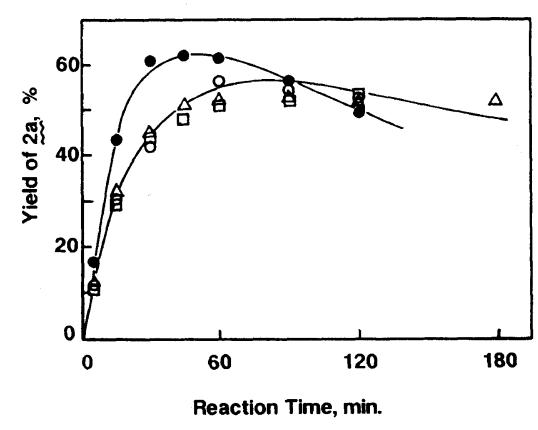

Figure 6. Effect of the additive on the yield of $\mathbf{2 a}$ in benzene at $75^{\circ} \mathrm{C}$ under nitrogen atmosphere. [DVB] $]_{0}=$ $[1 \mathrm{a}]_{0}=0.34 \mathrm{moll}^{-1} ;[\mathrm{AIBN}]=2.0 \times 10^{-3} \mathrm{moll}^{-1}$. [Catechol]: $\bigcirc, 0$ ppm; $\bigcirc, 100$ ppm; $\square, 500$ ppm; $\triangle, 2000$ ppm.

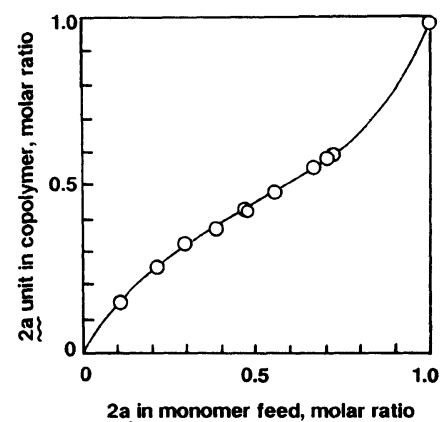

Figure 7. Copolymerization of 2a with MMA in benzene at $60^{\circ} \mathrm{C}$ under nitrogen atmosphere. $[2 \mathrm{a}]_{0}+[\mathrm{MMA}]_{0}=1.0$ mol1 $1^{-1} ;[\mathrm{AIBN}]=0.01 \mathrm{moll}^{-1} ;$ yields were less than $10 \%$. $[\eta]$ of copolymers in benzene at $30^{\circ} \mathrm{C}, 0.04-0.15 \mathrm{dl} \mathrm{g}^{-1}$.

although the addition of p-tert-butylcatechol in the reaction mixture decreased the reaction rate before $90 \mathrm{~min}$, the final yield of $\mathbf{2 a}$ increased.

\section{Copolymerization of $\mathbf{2 a}$ with $M M A$}

Since 2a has a polymerizable vinyl group, the copolymerization of $\mathbf{2 a}$ with methyl methacrylate was examined in benzene at $60^{\circ} \mathrm{C}$ in the presence of small amount of AIBN. The relationship between the monomer feed ratio and copolymer composition is shown in Figure 7 , where the copolymer yields were restricted below $10 \%$ to elucidate the accurate monomer reactivity ratio.

In Figure 7, the line was drawn based on the monomer reactivity ratio taking $r_{2 \mathrm{a}}=0.32$ and 
$r_{\mathrm{MMA}}=0.63$ calculated by means of Fineman-Ross method. From these monomer reactivity ratios, $Q_{2 \mathrm{a}}=0.69$ and $e_{2 \mathrm{a}}=-0.87$ were obtained based on the $Q-e$ rule. It is realized that $2 \mathbf{a}$ has the polymerizability and the monomer reactivity ratio of $\mathbf{2 a}$ to MMA is lower than that of $p$-methylstyrene to MMA, in which $r_{\mathrm{P}-\mathrm{Mst}}=0.44$ and $r_{\mathrm{MMA}}=0.40, Q_{\mathrm{P}-\mathrm{Mst}}=$ 1.10 , and $e_{\mathrm{p}-\mathrm{Mst}}=-0.63 .^{21}$ The intrinsic viscosities $[\eta]$ of these obtained copolymers were determined to be about 0.04 to $0.15 \mathrm{dl} \mathrm{g}^{-1}$ in benzene at $30^{\circ} \mathrm{C}$. The monomer $2 \mathbf{a}$ has the sulfide group, so that the sulfide group may act as a chain transfer reagent like as the diphenyl sulfide, $C_{\mathrm{tr}}=5.48 \times 10^{-3},{ }^{22}$ and diethyl sulfide, $C_{\mathrm{tr}}=2.7 \times 10^{-2} .^{23} 2 \mathrm{a}$ is similar to the molecular structure of diphenyl sulfide and diethyl sulfide, since $\mathbf{2 a}$ has the phenylthiomethylene group and consequently, the chain transfer constant of 2a may be almost the same as that of diphenyl sulfide and diethyl sulfide, which is much higher than that of benzene, $1.8 \times$ $10^{-6} .{ }^{23}$ Thus, $2 \mathrm{a}$ reduces the molecular weight of the copolymers.

\section{EXPERIMENTAL}

\section{Materials}

Thiophenol (Wako Pure Chem. Co.), p-toluenethiol (Aldrich Chem. Co.), and $m$-toluenethiol (Wako Pure Chem. Co.) were purified by distillation under reduced pressure under nitrogen atmosphere. 1,4-Divinylbenzene (Hokkou Chem. Co.) and methyl methacrylate (Wako Pure Chem. Co.) were also purified by distillation under reduced pressure under nitrogen atmosphere before use. AIBN was purified by recrystallization in hexane. Solvent benzene was used by distillation after being degassed with bubbling nitrogen for $2 \mathrm{~h}$.

\section{Preparation of $\mathbf{2 a}$}

In a four necked $200 \mathrm{ml}$ round bottom flask equipped with a three-way stopcock, equimolar amount of benzene solutions of thiophenol and DVB, a small amount of a benzene solution of
AIBN were charged by hypodermic syringe under nitrogen atmosphere. The initial concentrations of thiophenol, DVB and AIBN are $1.25 \mathrm{moll}^{-1}, 1.25 \mathrm{moll}^{-1}$, and $0.013 \mathrm{moll}^{-1}$, respectively. The addition reaction was carried out at $65^{\circ} \mathrm{C}$ for $90 \mathrm{~min}$ under nitrogen atmosphere.

The adduct $2 \mathbf{a}$ was isolated from the reaction mixture by two methods, column chromatography and distrillation under reduced pressure. By column chromatography, the reaction mixture was evaporated to remove the solvent, then developed in the column filled with Silica gel (Wako Gel, Q-23, 100-200 mesh) and carbon tetrachloride as an eluent, and then purified by distillation under reduced pressure. The distillation method was performed under reduced pressure adding small amounts of polymerization inhibitors, thiophenol or anhydrous ferric chloride after removed the benzene solvent. The two methods have almost the same efficiency for isolating 2a. The isolated yield of 2a, 4-vinyl-1-[(2-phenylthio)ethyl]benzene, was about $50 \%$; bp, $129-131^{\circ} \mathrm{C} / 0.04 \mathrm{mmHg}$; $n_{\mathrm{D}}^{25}=1.6211 ; m / z=240$; elementary analysis, Calcd: C, $80.0 \%$; H, 6.7\% ; S, 13.3\%. Found: C, $80.1 \%$; H, $6.6 \%$; S, $13.3 \%$.

\section{Preparation of $\mathbf{2 b}$}

4-Vinyl-1-[( $p$-tolylthio)ethyl]benzene, 2b was prepared in the same manner as above at $75^{\circ} \mathrm{C}$ for $115 \mathrm{~min}$. Yield, about $50 \%$; bp, $138^{\circ} \mathrm{C} / 0.06 \mathrm{mmHg} ; n_{\mathrm{D}}^{25}=1.6110$; Elementary analysis, Calcd: C, 80.3\%; H, 7.1\%; S, 12.6\%. Found: C, $80.0 \%$; H, 7.2\%; S, $12.5 \%$.

\section{Preparation of $\mathbf{2 c}$}

4-Vinyl-1-[( $m$-tolylthio)ethyl $]$ benzene, 2c, was also synthesized in the same method described above at $75^{\circ} \mathrm{C}$ for $120 \mathrm{~min}$. Yield, about $50 \%$; bp, $126-127^{\circ} \mathrm{C} /<10^{-3} \mathrm{mmHg}$; $n_{\mathrm{D}}^{25}=1.6115$; Sulfur content, Calcd, $12.6 \%$; Found, $12.6 \%$.

\section{Copolymerization of $\mathbf{2 a}$ with $M M A$}

The copolymerization of 2a with MMA was 
carried out at $60^{\circ} \mathrm{C}$ in a sealed glass tube, where definite amounts of 2a, MMA and AIBN with benzene were charged under nitrogen atmosphere. After polymerization, the reaction mixture was poured into a large amount of methanol to precipitate copolymers. The copolymers were washed with methanol several times and dried in vacuo overnight. The copolymer compositions were estimated from sulfur content of the copolymers by Schoeniger's method. ${ }^{24}$

\section{Measurements}

The reaction course was monitored by gas chromatography using a silicon GE SE-30, $2 \mathrm{~m}$ column and a flame ion detector. Column temperature, $250^{\circ} \mathrm{C}$; injection temperature, $300^{\circ} \mathrm{C}$; carrier gas flow, $\mathrm{N}_{2}, 32 \mathrm{ml} \mathrm{min}^{-1}$. The retention time was recorded in a minute: thiophenol, 0.3; DVB, 0.4; 2a, 6.3. Diphenyl sulfide was used as the external standard for the quantitative analysis of 2a was not observed by gas chromatography under this condition. By changing the G.C. conditions, column temperature, $260^{\circ} \mathrm{C}$; injection temperature,

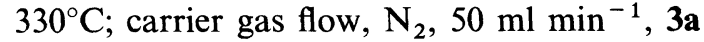
was detected in the retention time, $56.3 \mathrm{~min}$.

IR spectra were recorded with a Hitachi 260-50 infrared spectrometer and ${ }^{1} \mathrm{H}$ NMR was determined using a JEOL PMX-60si with the chemical shifts $\delta$ from TMS as $0 \mathrm{ppm}$. Mass spectra were recorded with a JMS D-300. The refractive index was measured by an Atago Abbe Refractometer 301 at $25^{\circ} \mathrm{C}$. Sulfur content of these adducts $\mathbf{2 a}, \mathbf{2 b}$, and $\mathbf{2 c}$ and the copolymers was determined by Schoeniger's method. ${ }^{24}$

\section{REFERENCES}

1. Xue-fen Li, M. Maeda, and T. Tsuruta, Makromol.
Chem., 182, 2445 (1981).

2. T. Narita, Y. Nitatori, T. Irie, and T. Tsuruta, Makromol. Chem., 177, 3255 (1979).

3. M. Maeda, Y. Nitadori, and T. Tsuruta, Makromol. Chem., 181, 2245 (1980).

4. T. Hatayama, K. Yamaguchi, A. Hirao, and S. Nakahama, Polym. Prepr. Jpn., 34, 190 (1985).

5. A. Hirao, K. Yamaguchi, K. Takenaka, K. Suzuki, S. Nakahama, and N. Yamazaki, Makromol. Chem., Rapid Commun., 3, 941 (1982).

6. A. Hirao, K. Takenaka, K. Yamaguchi, S. Nakahama, and N. Yamazaki, Polym. Commun., 24, 339 (1983)

7. T. Asahara, M. Seno, H. Kise, and H. Serita, Seisan Kenkyu, 24, 253 (1973).

8. Toray Co., Jpn. Patent, S-59-90846.

9. F. W. Stacey and J. F. Harris, Jr., J. Am. Chem. Soc., 85, 963 (1963).

10. A. A. Oswald, K. Griesbaum, B. E. Hudson, Jr., and J. M. Bregman, J. Am. Chem. Soc., 86, 2877 (1964).

11. K. Griesbaum, A. A. Oswald, and B. E. Hudson, Jr., J. Am. Chem. Soc., 85, 1969 (1963).

12. C. G. Screttas and M.-M. Screttas, J. Org. Chem., 43, 1064 (1978).

13. R. H. Pallen and C. Sivertz, Can. J. Chem., 35, 723 (1957).

14. M. Onyszchuk and C. Sivertz, Can. J. Chem., 33, 1034 (1955).

15. E. Kobayashi, T. Obata, S. Aoshima, and J. Furukawa, Polym. J., 22, 803 (1990).

16. J. Jiang, E. Kobayashi, T. Obata, and J. Furukawa, Polym. J., 22, 963 (1990).

17. S. C. Barton, R. A. Bird, and K. E. Russell, Can. J. Chem., 41, 2737 (1963).

18. J. P. Van Hook and A. V. Tobolsky, J. Am. Chem. Soc., 80, 779 (1958)

19. S. Oae, "Organic Sulfur Chemistry, Reaction Mechanism Section,” Kagaku Doujin, Tokyo, 1982, p 264.

20. T. Obata, M. Sc. Thesis, Science University of Tokyo, Noda (1990).

21. J. Brandrup and E. H. Immergut, "Polymer Handbook," 3rd ed, John Wiley and Sons, New York, 1989, pp II-200, II-269.

22. K. Tsuda, S. Kobayashi, and T. Otsu, Bull. Chem. Soc., Jpn., 38, 1517 (1965).

23. J. Brandrup and E. H. Immergut, "Polymer Handbook," 2nd ed, John Wiley and Sons, New York, 1975, p II-59.

24. W. Schoeniger, Mikrochim. Acta. 123 (1955). 